\title{
Erratum to: Emerging Infections and Pertinent Infections Related to Travel for Patients with Primary Immunodeficiencies
}

\author{
Kathleen E. Sullivan ${ }^{1} \cdot$ Hamid Bassiri ${ }^{2} \cdot$ Ahmed A. Bousfiha $^{3} \cdot$ Beatriz T. Costa-Carvalho ${ }^{4}$. \\ Alexandra F. Freeman ${ }^{5}$ David Hagin $^{6} \cdot$ Yu L. Lau ${ }^{7,8} \cdot$ Michail S. Lionakis $^{9}$ - Ileana Moreira ${ }^{10}$. \\ Jorge A. Pinto ${ }^{11}$ - M. Isabel de Moraes-Pinto ${ }^{12}$ - Amit Rawat ${ }^{13} \cdot$ Shereen M. Reda $^{14}$. \\ Saul Oswaldo Lugo Reyes ${ }^{15}$. Mikko Seppänen ${ }^{16} \cdot$ Mimi L. K. Tang ${ }^{17}$
}

Published online: 25 August 2017

(C) Springer Science+Business Media, LLC 2017

\section{Erratum to: J Clin Immunol.}

$$
\text { https://doi.org/ 10.1007/s10875-017-0426-2 }
$$

The original version of this article unfortunately contained a mistake. The author was incorrectly listed as Oyl Mikko Seppänen. It should have been Mikko Seppänen only. The original article was corrected.

The online version of the original article can be found at https://oi.org/ 10.1007/s10875-017-0426-2

Kathleen E. Sullivan

sullivank@email.chop.edu

Hamid Bassiri

bassiri@email.chop.edu

Ahmed A. Bousfiha

profbousfiha@gmail.com

Beatriz T. Costa-Carvalho

beacarvalho@terra.com.br

Alexandra F. Freeman

Freemaal@mail.nih.gov

David Hagin

Davidha@tlvmc.gov.il

Yu L. Lau

lauylung@hku.hk

Michail S. Lionakis

lionakism@mail.nih.gov

Ileana Moreira

ileanam@gmail.com

Jorge A. Pinto

jpinto@medicina.ufmg.br
M. Isabel de Moraes-Pinto

m.isabelmp@uol.com.br

Amit Rawat

rawatamit@yahoo.com

Shereen M. Reda

shereen.m.reda@gmail.com; Shereenreda@med.asu.edu.eg

Saul Oswaldo Lugo Reyes

dr.lugo.reyes@gmail.com

Mikko Seppänen

Mikko.Seppanen@hus.fi

Mimi L. K. Tang

mimi.tang@rch.org.au

Division of Allergy and Immunology, The Children's Hospital of Philadelphia, 3615 Civic Center Blvd, Philadelphia, PA 19104, USA

2 Division of Infectious Diseases and Center for Childhood Cancer Research, Children's Hospital of Philadelphia, Perelman School of Medicine at the University of Pennsylvania, 3501 Civic Center Boulevard, Philadelphia, PA 19104, USA 
3 Clinical Immunology Unit, Infectious Department, Hopital d'Enfant Abderrahim Harouchi, CHU Ibn Rochd, Laboratoire d'Immunologie Clinique, d'Inflammation et d'Allergie LICIA, Faculté de Médecine et de Pharmacie, Université Hassan II, Casablanca, Morocco

4 Department of Pediatrics, Federal University of São Paulo, Rua dos Otonis, 725, São Paulo, SP 04025-002, Brazil

5 NIAID, NIH, Building 10 Room 12C103, 9000 Rockville, Pike, Bethesda, MD 20892, USA

6 Division of Allergy and Immunology, Tel Aviv Sourasky Medical Center, Sackler Faculty of Medicine, University of Tel Aviv, 6 Weizmann St, 64239 Tel Aviv, Israel

7 Department of Paediatrics\&Adolescent Medicine, The University of Hong Kong, Rm106, 1/F New Clinical Building, Pok Fu Lam, Hong Kong

8 Queen Mary Hospital, 102 Pokfulam Road, Pok Fu Lam, Hong Kong

9 Fungal Pathogenesis Unit, Laboratory of Clinical Infectious Diseases, National Institute of Allergy \& Infectious Diseases (NIAID), National Institutes of Health (NIH), 9000 Rockville Pike, Building 10, Room 11C102, Bethesda, MD 20892, USA
10 Immunology Unit, Hospital de Niños Ricardo Gutiérrez, Gallo 1330, 1425 Buenos Aires, Argentina

11 Division of Immunology, Department of Pediatrics, Federal University of Minas Gerais, Av. Alfredo Balena 190, room \# 161, Belo Horizonte, MG 30130-100, Brazil

12 Division of Pediatric Infectious Diseases, Department of Pediatrics, Federal University of São Paulo, Rua Pedro de Toledo, $781 / 9^{\circ}$ andar, São Paulo, SP 04039-032, Brazil

13 Pediatric Allergy and Immunology, Department of Pediatrics, Advanced Pediatrics Centre, Postgraduate Institute of Medical Education and Research, Chandigarh, India

14 Pediatric Department, Faculty of Medicine, Ain Shams University, Cairo, Egypt

15 Immunodeficiencies Research Unit, National Institute of Pediatrics, Av Iman 1, Torre de Investigacion, Piso 9, Coyoacan, 04530 Mexico City, Mexico

16 Harvinaissairauksien yksikkö (HAKE), Rare Disease Center, Helsinki University Hospital (HUH), Helsinki, Finland

17 Murdoch Children's Research Institute, The Royal Children's Hospital, University of Melbourne, Melbourne, Australia 